\title{
CONGRUENCE PROPERTIES OF MULTIPLICATIVE FUNCTIONS ON SUMSETS AND MONOCHROMATIC SOLUTIONS OF LINEAR EQUATIONS
}

\author{
Christian Elsholtz, David S. Gunderson
}

\begin{abstract}
Letting $\Omega(n)$ denote the number of prime factors of $n$ counted with multiplicity, Rivat, Sárközy and Stewart (1999) proved a result regarding maximal cardinalities of sets $\mathcal{A}, \mathcal{B} \subset$ $\{1, \ldots, N\}$ so that for every $a \in \mathcal{A}$ and $b \in \mathcal{B}, \Omega(a+b)$ is even.

This paper extends their work in several directions. The role of $\lambda(n)=(-1)^{\Omega(n)}$ is generalized to all non-constant completely multiplicative functions $f: \mathbb{N} \rightarrow\{-1,1\}$. Rather than just $\Omega$ being even on $\mathcal{A}+\mathcal{B}$, we extend the result to all possible parities of $\Omega$ on $\mathcal{A}, \mathcal{B}$, and $\mathcal{A}+\mathcal{B}$. Furthermore, we prove that many such pairs $(\mathcal{A}, \mathcal{B})$ exist. Results from Ramsey theory and extremal graph theory are used.
\end{abstract}

Keywords: arithmetic Ramsey theory, multiplicative functions, extremal graph theory.

\section{Introduction}

Let $\Omega(n)$ denote the number of prime factors of $n$ counted with multiplicity, and let $\lambda(n)=(-1)^{\Omega(n)}$ be the Liouville function. Patterns in the values of this sequence have been extensively studied, for example because this function carries information about primes. This function also has been suggested to be a candidate for generating very good random sequences. In this paper we study more generally what type of patterns one would expect for an arbitrary non-constant completely multiplicative functions $f: \mathbb{N} \rightarrow\{-1,1\}$.

\subsection{Notation}

The integers are denoted by $\mathbb{Z}$ and the positive integers by $\mathbb{N}$. An interval of integers is denoted by $[a, b]=\{x \in \mathbb{Z}: a \leqslant x \leqslant b\}$. A subset of positive integers is denoted by a script capital, for example, $\mathcal{A} \subset[1, N]$. For sets $\mathcal{A}$ and $\mathcal{B}$, define $\mathcal{A}+\mathcal{B}=\{a+b: a \in \mathcal{A}, b \in \mathcal{B}\}$. For a positive integer $n, \Omega(n)$ denotes 
the number of prime factors of $n$ counted with multiplicity. All logarithms are to the base $e$. Throughout this paper, $c$ denotes a constant, with its meaning varying depending on the context. A function $f: \mathbb{N} \rightarrow \mathbb{Z}$ is said to be completely multiplicative if for all positive integers $m$ and $n, f(m n)=f(m) f(n)$.

For functions $f$ and $g: \mathbb{N} \rightarrow \mathbb{N}$, write $f=\Theta(g)$ if both $f=O(g)$ and $g=O(f)$, i.e., if there exist constants $c$ and $C$ so that for $n$ sufficiently large, $c g(n) \leqslant f(n) \leqslant$ $C g(n)$.

Extending the definition of the binomial coefficient $\left(\begin{array}{l}n \\ k\end{array}\right)$, for a positive real $x$ and non-negative integer $k$, let $\left(\begin{array}{l}x \\ k\end{array}\right)=\frac{x(x-1)(x-2) \ldots(x-k+1)}{k !}$.

\subsection{Results}

Rivat, Sárközy and Stewart [20] showed that large sets of positive integers $\mathcal{A}, \mathcal{B}$ could be found so that every element in the sumset $\mathcal{A}+\mathcal{B}$ has an even number of prime factors:

Theorem 1.1 (Rivat, Sarközy, Stewart). There exists a constant $c \geqslant \frac{1}{\log 3}$ so that for positive integers $N$ and $\ell$ with $\ell<c \log N$, there exist sets $\mathcal{A}, \mathcal{B} \subseteq[1, N]$ with $|\mathcal{A}|>\frac{N}{\ell 3^{\ell}}$ and $|\mathcal{B}|=\ell$ such that for all $a \in \mathcal{A}$ and $b \in \mathcal{B}, \Omega(a+b)$ is even.

Observe that Theorem 1.1 implies, e.g., the following: if $\ell$ is a constant, then $|\mathcal{A}|$ is of order of magnitude $N$, and if $\ell$ is about $\log \log N$, then $|\mathcal{A}| \approx \frac{N}{(\log N)^{\log 3} \log \log N}$. As $|\mathcal{B}|=\ell$ increases, $|\mathcal{A}|$ decreases, and one can work out that the cardinality of $\mathcal{A}$ and $\mathcal{B}$ is about the same when $\ell=\frac{\log N-2 \log \log N+O(1)}{\log 3}$.

However, in order to have a nontrivial result, one can assume that $\ell \leqslant \frac{\log N-\log \log N+O(1)}{\log 3}$, since otherwise even $\frac{N}{\ell^{\ell}}<1$ is true. For larger $\ell$, the theorem is easily true, for if $A=\{a\}$ then one can choose the set $\mathcal{B}=$ $\{b: \Omega(a+b)$ is even $\}$ of density $\frac{1}{2}$. Here and in the results below we assume that $|\mathcal{B}|=\ell<c \log N \leqslant|\mathcal{A}|$ for an appropriate constant $c$.

Some further results of related interests and methods can be found, for example in [13] and [7].

Our main results (Theorems 1.2, 1.3 and 1.4 below) generalize Theorem 1.1 in a number of ways, the first of which also contains a slight refinement of the constants involved:

Theorem 1.2. Let $f: \mathbb{N} \rightarrow\{-1,1\}$ be a non-constant completely multiplicative function. Let $p_{0}$ be the least prime so that $f\left(p_{0}\right)=-1$, and let $\ell, m \in \mathbb{Z}^{+}$. Let $\eta \in\{-1,1\}$. If $N>p_{0}\left(2^{\ell}(m-1)+1\right)$, there exist $\mathcal{A}, \mathcal{B} \subset[1, N]$, with $\ell$ and $m$ elements, respectively, and for all $a \in \mathcal{A}, b \in \mathcal{B}, f(a+b)=\eta$.

To state the next generalization, some notation is convenient. If $f$ is a nonconstant completely multiplicative function, define

$$
c_{f}^{-}=\frac{1}{2}\left(1-\prod_{\substack{p \\ f(p)=-1}}\left(1-\frac{2}{p+1}\right)\right),
$$


and where $c_{f}^{-}=\frac{1}{2}$ if the infinite product diverges, and define $c_{f}^{+}=1-c_{f}^{-}$. Note that $c_{f}^{-}, c_{f}^{+} \in(0,1)$.

Theorem 1.3. Let $f: \mathbb{N} \rightarrow\{-1,1\}$ be a non-constant completely multiplicative function and let $\left(\eta_{1}, \eta_{2}, \eta_{3}\right) \in\{-1,1\}^{3}$. Define for $i=1,2$

$$
c_{i}=\left\{\begin{array}{ll}
c_{f}^{-} & \text {if } \eta_{i}=-1 \\
c_{f}^{+} & \text {if } \eta_{i}=+1
\end{array},\right.
$$

and put $c=\min \left\{c_{1}, c_{2}\right\}$.

For $N \geqslant N(f)$ sufficiently large, and $\ell<c \log N$, there exists a constant $c_{1}^{\prime}=c_{1}^{\prime}(f) \in(0,1)$, and there exist $\mathcal{A} \subset[1, N]$ and $\mathcal{B} \subset[1, N]$, such that

(i) for all $a \in \mathcal{A}, b \in \mathcal{B}, f(a)=\eta_{1}, f(b)=\eta_{2}$, and $f(a+b)=\eta_{3}$;

(ii) $|\mathcal{B}|=\ell$;

(iii) $|\mathcal{A}|>\left(c_{1}^{\prime}\right)^{\ell} N$.

Theorem 1.4. Let $f, \eta_{i}$ and $c_{i}$ be as in the previous theorem. Let $v$ be the least integer such that

(i) $f\left(n_{1}\right)=f\left(n_{1}+1\right)=1$,

(ii) $f\left(n_{2}\right)=1, f\left(n_{2}+1\right)=-1$,

(iii) $f\left(n_{3}\right)=-1, f\left(n_{3}+1\right)=1$

have a solution with $n_{i} \leqslant v$. Let

$$
\begin{aligned}
& m:=\left|\left\{j \leqslant N: f(j)=\eta_{1}\right\}\right|=(1+o(1)) c_{1} N, \\
& n:=\left|\left\{j \leqslant N: f(j)=\eta_{2}\right\}\right|=(1+o(1)) c_{2} N,
\end{aligned}
$$

(compare Lemma 3.4). Moreover, let

$$
C_{3}=\frac{1}{138,000(v+1)^{3}} N^{2} .
$$

Then the number of such pairs $(\mathcal{A}, \mathcal{B})$ with $s=|\mathcal{A}|$ and $t=|\mathcal{B}|$ and $f(a+b)=\eta_{3}$ is at least

$$
\left(\begin{array}{c}
m \\
s
\end{array}\right)\left(\begin{array}{c}
\frac{n}{\left(\begin{array}{c}
m \\
s
\end{array}\right)}\left(\begin{array}{c}
C_{3} / n \\
s
\end{array}\right) \\
t
\end{array}\right)
$$

Moreover, assuming that $s$ and $t$ are both $o\left(N^{3 / 4}\right)$, then the expression above can be simplified to:

$$
\frac{1}{\left(138,000(v+1)^{3} c_{1} c_{2}\right)^{s t}}\left(\begin{array}{c}
m \\
s
\end{array}\right)\left(\begin{array}{l}
n \\
t
\end{array}\right) .
$$

Here the actual value of the constant $138,000(v+1)^{3}$ can be somewhat improved upon. The emphasis here is, that the constant depends on $f$ in a "reasonable", say polynomial, way. Below we will discuss several approaches, where for example a straightforward application of Szemerédi-type results would not allow to specify 
the constants in the same way. In the present form these constants show that the size and number of the "good" sets essentially depend on the size of the smallest prime with $f(p)=-1$, and (via $c_{f}^{-}$) on the frequency of these primes.

The last expression means that in the case of a fixed $s$ and $t$ a positive proportion of all choices of $s$ out of $m$ elements of and $t$ out of $n$ elements have the requested property.

The proofs of Theorem 1.2 and of Theorems 1.3 and 1.4 are concluded in Sections 5 and 8 , respectively.

To see that Theorem 1.2 generalizes Theorem 1.1, first note that the Liouville $\lambda$-function defined by $\lambda(n)=(-1)^{\Omega(n)}$ is indeed one example of a non-constant completely multiplicative function, and so Theorem 1.1 is included in the case $f=\lambda$, where $f(a+b)=\eta_{3}=1=(-1)^{\Omega(a+b)}$ since every $\Omega(a+b)$ is even. Note also that in Theorems 1.3 and 1.4 , the sets of integers $\mathcal{A}, \mathcal{B} \subseteq[1, N]$ can in addition have the values of the function at each $a$ and $b$ prescribed. In Ramseytheoretic terms, not just the sums are monochromatic, but also all the $a$ 's are of a prescribed colour and all the $b$ 's are of a prescribed colour, and for every possible colour pattern, sets $\mathcal{A}, \mathcal{B}$ exist that witness that pattern. Also, rather than producing one pair $\mathcal{A}, \mathcal{B}$, many such pairs are guaranteed in Theorem 1.4.

Theorem 1.3 implies the following corollaries on triples $\left(a, \frac{a+b}{2}, b\right)$ in arithmetic progression:

Corollary 1.5. Let $f: \mathbb{N} \rightarrow\{-1,1\}$ be a non-constant completely multiplicative function and let $\left(\eta_{1}, \eta_{2}, \eta_{3}\right) \in\{-1,1\}^{3}$. There exists $N_{0}=N_{0}(f)$ so that for every $N \geqslant N_{0}$, there exists a positive constant $c_{2}=c_{2}(f)$ and there exist $\mathcal{A}^{\prime}, \mathcal{B}^{\prime} \subset[1,2 N]$ such that

(i) for all $a \in \mathcal{A}^{\prime}, b \in \mathcal{B}^{\prime}, f(a)=\eta_{1}, f(b)=\eta_{2}$, and $f\left(\frac{a+b}{2}\right)=\eta_{3}$;

(ii) $\left|\mathcal{B}^{\prime}\right|=\ell$;

(iii) $\left|\mathcal{A}^{\prime}\right|>c_{2}^{\ell} N$.

Proof. Let $\mathcal{A}$ and $\mathcal{B}$ be guaranteed by Theorem 1.3, and double all elements: $\mathcal{A}^{\prime}=\{2 a: a \in \mathcal{A}\}$ and $\mathcal{B}^{\prime}=\{2 b: b \in \mathcal{B}\}$. Then $f\left(a^{\prime}\right)=f(2 a)=f(2) f(a)$, $f\left(b^{\prime}\right)=f(2 b)=f(2) f(b)$, and $f\left(\frac{a^{\prime}+b^{\prime}}{2}\right)=f\left(\frac{2 a+2 b}{2}\right)=f(a+b)$. Since Theorem 1.3 allows all possible sign combinations one also gets all possible sign combinations with $\mathcal{A}^{\prime}$ and $\mathcal{B}^{\prime}$. In analogy to Theorem 1.3 there are many such copies.

Theorem 1.3, applied in the range when $\mathcal{A}$ and $\mathcal{B}$ have about the same cardinality, i.e., with $\ell$ of order of magnitude $\log N$, gives:

Corollary 1.6. Let $f$ be a non-constant multiplicative function and let $\left(\eta_{1}, \eta_{2}, \eta_{3}\right) \in$ $\{-1,1\}^{3}$. For any positive constant $c_{3}<\frac{1}{\left|\log c^{-}\right|}$, and for sufficiently large $N$, there exist $\mathcal{A}$ and $\mathcal{B}$ such that $|\mathcal{A}|=|\mathcal{B}| \geqslant c_{3} \log N$ and for all $a \in \mathcal{A}, b \in \mathcal{B}, f(a)=\eta_{1}$, $f(b)=\eta_{2}$, and $f(a+b)=\eta_{3}$.

In the course of proving the results above, we also prove the following theorem, which may be of independent interest since it appears to be the first such result with explicit constants: 
Theorem 1.7. Let $a, b, c$ be nonzero integers with $a+b+c=0$. Let $W=W(a, b, c)$ denote the least integer such that for any 2-colouring $\chi:[1, W] \rightarrow\{$ red, blue $\}$, there exists a monochromatic solution of $a x+b y+c z=0$ in positive integers $x, y, z$, i.e., $\chi(x)=\chi(y)=\chi(z)$. Then $W(a, b, c) \leqslant 32 \max \{|a|,|b|,|c|\}+1$. For $N$ sufficiently large there are at least $\frac{N^{2}}{4 W^{3}} \geqslant \frac{N^{2}}{138,000|b|^{3}}$ monochromatic solutions of $a x+b y+c z=0$, with $x, y, z \leqslant N$.

The remainder of this paper is organized as follows. Section 2 is about completely multiplicative functions; this section contains no new results but establishes motivation and perspective. In Section 3, methods from analytic number theory are used to find hypotheses under which a completely multiplicative function $f$ takes the values +1 and -1 each about half the time. Notation for bipartite graphs is given in Section 4. A result by Thomason on bipartite Ramsey numbers is used to prove Theorem 1.2 in Section 5. In Section 6, some arithmetic Ramsey theory is reviewed that is needed to yield $\Theta\left(N^{2}\right)$ many triples $x_{1}, x_{2}, x_{3} \in[1, N]$ with $x_{1}+x_{2}=x_{3}$ and $f\left(x_{i}\right)=\eta_{i}$ as required.

Some required extremal graph theory is given in Section 7, where an explicit version of a result by Kövari, Sós, and Turán is given. Finally, in Section 8, the proofs of Theorems 1.3 and 1.4 is assembled, where a bipartite graph is defined, where edges correspond to the triples mentioned above, and this graph is shown to have sufficiently many edges to yield our result. The proof of Corollary 1.6 is also in Section 8.

\section{Completely multiplicative functions}

One reason why the function $\Omega$ is of interest is that the Liouville $\lambda$-function defined by $\lambda(n)=(-1)^{\Omega(n)}$ behaves in many aspects like a random sequence. Many authors have investigated those patterns that occur in the values of $\lambda$. For example, Chowla [4] conjectured that for any fixed $k$ and any pattern $\left(\eta_{1}, \ldots, \eta_{k}\right) \in\{-1,1\}^{k}$ there are infinitely many $n$ such that for $1 \leqslant i \leqslant k, \lambda(n+i)=\eta_{i}$. Conditionally, assuming Schinzel's Hypothesis $H$ (which generalizes the twin prime conjecture; see [23]), Cassaigne, Ferenczi, Mauduit, Rivat and Sárközy [3] proved this any fixed $k$. Unconditionally, Hildebrand [15] proved this for $k=3$, but it seems very difficult to extend Hildebrand's result to $k=4$, as problems related to the "parity problem" in sieve theory appear to come in.

As a partial result in this direction Buttkewitz and Elsholtz [2] proved for a completely multiplicative function $f: \mathbb{N} \rightarrow\{-1,1\}$, with at least two primes $p_{1}, p_{2}$ such that $f\left(p_{1}\right)=f\left(p_{2}\right)=-1$, and assuming that $f \neq f_{ \pm}$, where

$$
f_{ \pm}(n)= \begin{cases}( \pm 1)^{k}, & \text { if } n=3^{k} m, m \equiv 1 \bmod 3 \\ -( \pm 1)^{k}, & \text { if } n=3^{k} m, m \equiv 2 \bmod 3\end{cases}
$$

that each of the 16 possible sign patterns $( \pm 1, \pm 1, \pm 1, \pm 1)$ occurs as values along arithmetic progressions of size $k=4$, namely as $(f(n), f(n+d), f(n+2 d)$, $f(n+3 d))$, infinitely often. 
For a completely multiplicative function $f: \mathbb{N} \rightarrow\{-1,1\}$ with $f(2)=-1$ and for positive integers $k$ and $d$, Cassaigne, Ferenczi, Mauduit, Rivat and Sárközy [3] proved

$$
\left|\sum_{n \leqslant N} f(n) f(n+d) \cdots f(n+2 k d)\right| \leqslant\left(1-c_{k}\right) N+O(\log N),
$$

where

$$
c_{k}= \begin{cases}\left(1-\frac{2}{3(2 k+1)}\right) & \text { if } d \text { is even } \\ \left(1-\frac{2}{3(k+1)}\right) & \text { if } d \text { is odd }\end{cases}
$$

and the $O$-constant depends on $k$ and $d$ only.

Harman, Pintz and Wolke [14] proved that the sequence $\langle\lambda(n)\rangle$ contains many sign changes, but Graham and Hensley [12] proved that there are not too many: for all $\varepsilon>0$ and all $N>N_{\varepsilon}$,

$$
-\frac{1}{3}+O\left(\frac{\log N}{N}\right)<\frac{1}{N} \sum_{n \leqslant N} \lambda(n) \lambda(n+1)<1-\frac{1}{(\log N)^{7+\varepsilon}} .
$$

Croot [5] showed for a completely multiplicative function $f: \mathbb{N} \rightarrow\{-1,1\}$, if $\sum_{n \leqslant N} f(n)=o(N)$, then the sequence $\langle f(n)\rangle$ contains at least $\frac{c N}{\exp (7(\log \log N) \sqrt{\log N})}$ sign changes.

Added in proof: Very recently Matomäki, Radziwiłł [17] and these authors jointly with Tao [18] proved several breakthrough results which for example includes the following (Corollary 2 of [17]): For every completely multiplicative function $f: \mathbb{N} \rightarrow[-1,1]$ such that $f(n)<0$ for some $n$, and for every integer $h \geqslant 1$, there exists $\delta(h)>0$ such that $\frac{1}{N}\left|\sum_{n \leqslant N} f(n) f(n+h)\right| \leqslant 1-\delta(h)$ for all sufficiently large $N$, which means in particular that there is a positive proportion of sign changes.

\section{Pseudorandom properties of completely multiplicative functions}

The sequence $\langle\lambda(n)\rangle$ takes the value +1 asymptotically half the time (and so also the value -1 half the time) and behaves in some respects like a random sequence. In this section (Lemma 3.3) conditions on completely multiplicative functions $f$ are examined so that the sequence $\langle f(n)\rangle$ also takes the value +1 half the time, and determine the density of the +1 or -1 values in the other cases.

For $f: \mathbb{N} \rightarrow\{1,-1\}$, define $S^{+}(f)=\{n \in \mathbb{N}: f(n)=1\}$ and $S^{-}(f)=$ $\{n \in \mathbb{N}: f(n)=-1\}$. When it causes no confusion, these two sets are referred to as simply $S^{+}$and $S^{-}$respectively. A set $S$ of positive integers has upper density $d$, if $\limsup _{N \rightarrow \infty} \frac{S \cap[1, N]}{N}=d$. An elementary but critical observation is:

Lemma 3.1. Let $f: \mathbb{N} \rightarrow\{1,-1\}$ be a non-constant completely multiplicative function. Then both sets $S^{+}(f)$ and $S^{-}(f)$ have positive upper density. 
Proof. Let $p_{0}$ denote the least integer (indeed a prime) with $f\left(p_{0}\right)=-1$. At least one of the sets $S^{+}$or $S^{-}$must have positive upper density; say $S^{+}$has upper density at least $\alpha$. Then the map $g: S^{+} \rightarrow S^{-}$defined by $g(n)=p_{0} n$ shows that $S^{-}$has upper density at least $\frac{\alpha}{p_{0}}$. Similarly, if $S^{-}$has positive upper density it follows that $S^{+}$has positive upper density.

The densities of $S^{+}(f)$ and $S^{-}(f)$ are completely determined by the values of $f$ on prime numbers and can be stated explicitly. (For related results, even with error terms see [6].) To prove the main lemma (Lemma 3.4) of this section about the densities of $S^{+}$and $S^{-}$, two results are needed, a theorem by Wirsing [29] (see also, e.g., [25, p. 336]) and a lemma about "balanced" $S^{+}$and $S^{-}$.

Theorem 3.2 (Wirsing). Let $g$ be a real multiplicative function with values in the real interval $[-1,1]$. Then

$$
\lim _{N \rightarrow \infty} \frac{1}{N} \sum_{n \leqslant N} g(n)=\prod_{p \text { prime }}\left(1-\frac{1}{p}\right) \sum_{\nu=0}^{\infty} \frac{g\left(p^{\nu}\right)}{p^{\nu}},
$$

where the infinite product is to be taken as zero when it is divergent.

Lemma 3.3. Let $f: \mathbb{N} \rightarrow\{1,-1\}$ be a completely multiplicative function. The following three conditions are equivalent.

$$
\begin{array}{r}
\sum_{\substack{p \text { prime } \\
f(p)=-1}} \frac{1}{p} \text { diverges. } \\
\sum_{\substack{n \leqslant N \\
f \leqslant N}} f(n)=o(N) . \\
\sum_{\substack{n \leqslant N \\
f(n)=1}} 1 \sim \sum_{\substack{n \leqslant N \\
f(n)=-1}} 1 \sim \frac{1}{2} N .
\end{array}
$$

Proof of Lemma 3.3. $(2) \Leftrightarrow(3)$ : If $f(p)=1$, then

$$
\left(1-\frac{1}{p}\right) \sum_{\nu=0}^{\infty} \frac{f\left(p^{\nu}\right)}{p^{\nu}}=\frac{p-1}{p} \sum_{\nu=0}^{\infty} \frac{1}{p^{\nu}}=1 .
$$

If $f(p)=-1$, then

$$
\left(1-\frac{1}{p}\right) \sum_{\nu=0}^{\infty} \frac{f\left(p^{\nu}\right)}{p^{\nu}}=\frac{p-1}{p} \cdot \frac{1}{1+\frac{1}{p}}=1-\frac{2}{p+1} .
$$

So

$$
\prod_{p \text { prime }}\left(1-\frac{1}{p}\right) \sum_{\nu=0}^{\infty} \frac{f\left(p^{\nu}\right)}{p^{\nu}}=\prod_{\substack{p \text { prime } \\ f(p)=-1}}\left(1-\frac{2}{p+1}\right)
$$


which tends to 0 if and only if $\sum_{\substack{p \text { prime } \\ f(p)=-1}} \frac{1}{p}$ diverges. By Wirsing's theorem (Theorem 3.2), this is equivalent to $\sum_{n \leqslant N}^{f(p)=-1} f(n)=o(N)$.

$(3) \Rightarrow(4)$ : Since one of

$$
\sum_{\substack{n \leqslant N \\ f(n)=1}} 1 \quad \text { or } \quad \sum_{\substack{n \leqslant N \\ f(n)=-1}} 1
$$

must be at least $\frac{1}{2} N,(3)$ implies that both must be asymptotically $\frac{1}{2} N$.

$(4) \Rightarrow(3):$ If

$$
\sum_{\substack{n \leqslant N \\ f(n)=1}} 1 \sim \frac{1}{2} N \quad \text { and } \quad \sum_{\substack{n \leqslant N \\ f(n)=-1}} 1 \sim \frac{1}{2} N
$$

then

$$
\sum_{n \leqslant N} f(n)=\sum_{\substack{n \leqslant N \\ f(n)=1}} 1-\sum_{\substack{n \leqslant N \\ f(n)=-1}} 1=o(N)
$$

Lemma 3.4. Let $f: \mathbb{N} \rightarrow\{1,-1\}$ be a non-constant completely multiplicative function, and put

$$
c=\prod_{\substack{p \\ f(p)=-1}}\left(1-\frac{2}{p+1}\right)
$$

where the infinite product is to be taken as zero when it is divergent. The density of $S^{+}$is

$$
c^{+}:=\lim _{N \rightarrow \infty} \frac{1}{N} \sum_{\substack{n \leqslant N \\ f(n)=+1}} 1=\frac{1+c}{2},
$$

and the density of $S^{-}$is

$$
c^{-}:=\lim _{N \rightarrow \infty} \frac{1}{N} \sum_{\substack{n \leqslant N \\ f(n)=-1}} 1=\frac{1-c}{2} .
$$

Proof. From the proof of Lemma 3.3, it follows that the mean value of the function $f$ is

$$
\lim _{n \rightarrow N} \frac{1}{N} \sum_{n \leqslant N} f(n)=c=\prod_{\substack{p \text { prime } \\ f(p)=-1}}\left(1-\frac{2}{p+1}\right) .
$$

With $c^{+}+c^{-}=1$ and $c^{+}-c^{-}=c$ the lemma follows.

Note that the case of Lemma 3.4 where $c^{+}=c^{-}=\frac{1}{2}$, (i.e., where $c=0$ ), is dealt with in Lemma 3.3 and is of independent interest. This case sheds light on Croot's condition on $f$ cited above, and it is the most important case for the study of pseudorandom numbers. 


\section{Bipartite graphs}

To prove Theorems 1.2 and 1.3, bipartite graphs are used. For a set $X$ and positive integer $s$, let $[X]^{s}=\{S \subseteq X:|S|=s\}$. A graph $G$ is a pair $(V, E)=(V(G), E(G))$, where $V$ is a set and $E \subseteq[V]^{2}$. An element of $V$ is called a vertex, and an element of $E$ is called an edge. (Note, that under this definition, a graph has no loops nor multiple edges.) A graph $G$ is called bipartite if there is a partition of the vertices $V=V_{1} \cup V_{2}$ so that all edges contain a vertex from each of $V_{1}$ and $V_{2}$, that is, $E(G) \subseteq\left\{\{x, y\}: x \in V_{1}, y \in V_{2}\right\}$. The sets $V_{1}$ and $V_{2}$ are called partite sets, and such a graph is often then denoted $G=\left(V_{1} \cup V_{2}, E\right)$.

The graph $K_{a, b}$ is the bipartite graph $\left(V_{1} \cup V_{2}, E\right)$, where $\left|V_{1}\right|=a,\left|V_{2}\right|=b$, and $E(G)=\left\{\{x, y\}: x \in V_{1}, y \in V_{2}\right\}$. Note that under this definition, $K_{a, b}=K_{b, a}$.

If one needs to consider the partition $V_{1} \cup V_{2}$ as an ordered partition, call such a bipartite graph oriented, and denote such a graph with an arrow above; for example, $\overrightarrow{K_{a, b}}$ is the complete bipartite graph whose first partite set with $\left|V_{1}\right|=a$ vertices may be considered on the left. For two oriented bipartite graphs $\vec{G}=\left(V_{1} \cup V_{2}, E\right)$ and $\vec{H}=\left(W_{1} \cup W_{2}, F\right), \vec{H}$ is a subgraph of $\vec{G}$ if and only if $W_{1} \subseteq V_{1}, W_{2} \subseteq V_{2}$, and $F \subseteq E$.

\section{Proof of Theorem 1.2}

The proof relies on a result of Thomason [26] on bipartite Ramsey numbers. In some cases the bound is sharp.

Theorem 5.1. Let $b(s, t)$ denote the smallest integer $r$ such that every colouring of $K_{r, r}$ with red and blue contains a red $\overrightarrow{K_{s, t}}$ or a blue $\overrightarrow{K_{t, s}}$. Then $b(s, t) \leqslant 2^{s}(t-1)+1$ holds.

Proof of Theorem 1.2: Let $f$ be a non-constant completely multiplicative function with $p_{0}$ the least prime so that $f\left(p_{0}\right)=-1$. Fix $\ell \in \mathbb{Z}^{+}$, and $\eta \in\{-1,1\}$. Let $N>p_{0}\left(2^{\ell}(m-1)+1\right)$.

Consider the (oriented) complete bipartite graph $\vec{G}$ on partite sets $V_{1}$ and $V_{2}$ (and all $\left|V_{1}\right| \cdot\left|V_{2}\right|$ edges). Define the 2-colouring $\chi: E(G) \rightarrow\{-1,1\}$ by $\chi(\{i, j\})=f(i+j)$. Let $\vec{H}=(X, Y, F)$ be the subgraph of $\vec{G}$ induced by the integers smaller than $N / p_{0}$. Then each partite set, i.e. $X$ and $Y$, of $H$ has at least $2^{\ell}(m-1)+1$ vertices.

By Theorem 5.1, there exists a monochromatic copy of $\overrightarrow{K_{\ell, m}}$ or $\overrightarrow{K_{m, \ell}}$ in $H$. If the edges of this copy of $\overrightarrow{K_{\ell, m}}$ or $\overrightarrow{K_{m, \ell}}$ have colour $\eta$ then the proof is complete, with $X=\mathcal{A}$ and $Y=\mathcal{B}$, (or vice versa). If the edges of this copy of $K_{s, t}$ have colour $-\eta$ (the wrong colour), then the sets formed by multiplying $p_{0}$ in the respective complements of $X$ and $Y$ produce the desired monochromatic subgraph. 


\section{Results from arithmetic Ramsey theory}

In the early 1950's, Roth [21, 22] showed that for any $\varepsilon>0$, for sufficiently large $n$, any subset of $[1, n]$ with density $\varepsilon$ (i.e., with $\varepsilon n$ elements) contains a 3 -term arithmetic progression. This was later generalized to arbitrarily long arithmetic progressions by Szemerédi [24]. In 1959, Varnavides [28] generalized Roth's result by showing that for $\varepsilon>0$, there exists a constant $C=C(\varepsilon)$ so that for sufficiently large $N$, any subset of $[1, N]$ with density $\varepsilon$ contains at least $C N^{2}$ many 3 -term arithmetic progressions. Varnavides' idea was simple: apply Roth's theorem to each of the $\Theta\left(N^{2}\right)$ arithmetic progressions of length $n$ in $[1, N]$. The Varnavides argument also applies with Roth's theorem replaced by Szemerédi's theorem on $k$-term progressions.

One extension of the Varnavides idea is contained in the following special case of a powerful result due to Frankl, Graham, and Rödl [9, Thm 2]:

Theorem 6.1 (Frankl, Graham, Rödl). Let $a, b, c \in \mathbb{Z} \backslash\{0\}$ satisfy $a+b+c=0$. For all $\varepsilon>0$, there are constants $C=C(\varepsilon, a, b, c)>0$ and $N_{0}=N_{0}(\varepsilon, a, b, c)$ so that for any $N \geqslant N_{0}$, if $S \subseteq[1, N]$ satisfies $|S| \geqslant \varepsilon N$, then

$$
a x+b y+c z=0
$$

has at least $C N^{2}$ solutions $(x, y, z)$ in $S$.

Since the number of solutions to (5) with any two of $x, y, z$ the same is only linear in $N$, one can assume that $x, y$ and $z$ are pairwise distinct. With $a=c=1$ and $b=-2$, Theorem 6.1 is the Varnavides result.

In applications, where one is only concerned that the constant $C(\varepsilon, a, b, c)$ is positive, the theorem above is perfectly suitable. But since the constants coming from the above density result are very tiny, we decided to work out more reasonable constants. For this we establish bounds for a variant of van der Waerden's result, which may be of independent interest.

Instead of using a density result, one can apply the same Varnavides technique with a Ramsey-type (colouring) result. (This idea is also outlined in [9, bottom of p. 247].)

Recall van der Waerden's theorem:

Theorem 6.2 (van der Waerden, [27]). For all positive integers $k$ and $r$, there exists an integer $W(k ; r)$ such that for all $N \geqslant W(k ; r)$, if $[1, N]=C_{1} \cup C_{2} \cup \cdots \cup C_{r}$, then some $C_{i}$ contains an arithmetic progression of length $k$.

The required variant below uses only two colours, but for "weighted arithmetic progressions", i.e., solutions of the homogeneous linear equation $a x+b y+c z=0$, where $a+b+c=0$.

While the constant involved is not the best constant one may achieve, it is (to the best of our knowledge) the first explicit result of its kind. We tried to achieve a reasonable constant, while keeping the presentation simple. For any particular set of parameters $(a, b, c)$ one can certainly work out an improvement. 
Moreover, even in the case of arithmetic progressions, $(a=c=1, b=-2)$, the exact constant is not known. At the Erdôs conference in Budapest 1999, Graham offered $\$ 100$ for the solution of the following open problem (see [19]): it is expected that there is a positive constant $c^{\prime}$ so that for any 2-colouring of $[1, N]$ the minimum number $f(N)$ of monochromatic arithmetic progressions of length 3 is $f(N)=\left(c^{\prime}+o(1)\right) N^{2}$. It was thought that possibly $c^{\prime}=\frac{1}{16}$, coming from random colourings; however, Parrilo, Robertson and Paracino [19] proved that the minimum number of monochromatic arithmetic progressions is between $\frac{117+o(1)}{2192} N^{2}$ and $\frac{1675+o(1)}{32786} N^{2}$.

Proof of Theorem 1.7. Without loss of generality, suppose that $b<0<a \leqslant c$, so $\max \{|a|,|b|,|c|\}=|b|$.

Let us assume there is no monochromatic solution $(x, y, z)$ of equation (5).

Observe that for solutions of (5) generally $z=\frac{-a x-b y}{c}$, and if $y=x+t c$ (say), then $z=\frac{-a x-b x-b t c}{c}=x-t b$, since $a+b+c=0$.

Claim: There is a triple $(x, y, z)$ being a solution of equation $(5)$ with $\chi(x)=$ $\chi(y) \neq \chi(z)$.

Case 1: If $\chi(x)=\chi(x+c)$, then we set $t=1, y=x+c$. Since by assumption there is no monochromatic solution $(x, y, z)$ of equation $(5), z=x-b$ is of the other colour, which proves the claim.

Case 2: So we can assume that $\chi(x) \neq \chi(x+c)$. If $\chi(x)=\chi(x+2 c)$, then we set $t=2, y=x+2 c$. As above $z=x-2 b$ is of the other colour, and we are done.

Case 3: Here we can assume that $\chi(x) \neq \chi(x+c)$ and $\chi(x) \neq \chi(x+2 c)$, so that $\chi(x+c)=\chi(x+2 c)$. We then study the triple $(x+c, y=x+2 c, z=$ $\left.\frac{-a(x+c)-b(x+2 c)}{c}=x-a-2 b\right)$, which is a solution of equation (5). Again, as there is no such monochromatic solution, $z$ is of the other colour and we are done.

To summarize these three cases: One of the triples $(x, x+c, x-b),(x, x+$ $2 c, x-2 b),(x+c, x+2 c, x-a-2 b)$ is of the required type, and for later reference we write this as

$$
\left(x+\varepsilon_{1} c, x+\varepsilon_{2} c, x-a \varepsilon_{1}-b \varepsilon_{2}\right),
$$

where $\varepsilon_{i} \in\{0,1,2\}$. In other words, each interval $[x, x+2|b|]$ of length $2|b|+1$ contains a pattern of the required type.

There are six different colourings in total, two coming from each of the cases $\chi(x)=\chi(x+c) \neq \chi(x-b), \chi(x)=\chi(x+2 c) \neq \chi(x-2 b)$ and $\chi(x+c)=$ $\chi(x+2 c) \neq \chi(x-a-2 b)$. Hence, if there are seven different blocks of length $2|b|+1$, then there must be two blocks with exactly the same colour pattern. We will now study intervals of length $2|b|+1$ with the difference between these intervals being a suitable multiple of $c$. In order to avoid overlap, observe that $2|b|+1=2(a+c)+1 \leqslant 5 c$.

Examine the seven triples

$$
\left(x+\varepsilon_{1} c+m c, x+\varepsilon_{2} c+m c, x-a \varepsilon_{1}-b \varepsilon_{2}+m c\right),
$$


where $m \in\{0,5,10,15,20,25,30\}$. Assume that for $m_{1}$ and $m_{2}$ (say) the colour pattern of

$$
\left(x_{1}, y_{1}, z_{1}\right)=\left(x+\varepsilon_{3} c+m_{1} c, x+\varepsilon_{4} c+m_{1} c, x-a \varepsilon_{3}-b \varepsilon_{4}+m_{1} c\right),
$$

and

$$
\left(x_{2}, y_{2}, z_{2}\right)=\left(x+\varepsilon_{3} c+m_{2} c, x+\varepsilon_{4} c+m_{2} c, x-a \varepsilon_{3}-b \varepsilon_{4}+m_{2} c\right)
$$

is the same. The triple

$$
\left(x_{1}, y_{2}, z^{\prime}\right)=\left(x+\varepsilon_{3} c+m_{1} c, x+\varepsilon_{4} c+m_{2} c, z^{\prime}\right)
$$

with $z^{\prime}=x-a \varepsilon_{3}-b \varepsilon_{4}-a m_{1}-b m_{2}$ shows that $z^{\prime}$ cannot be of the first colour, and the triple

$$
\left(z_{1}, z_{2}, z^{\prime}\right)=\left(x-a \varepsilon_{3}-b \varepsilon_{4}+m_{1} c, x-a \varepsilon_{3}-b \varepsilon_{4}+m_{2} c, z^{\prime}\right)
$$

with the same $z^{\prime}=x-a \varepsilon_{3}-b \varepsilon_{4}-a m_{1}-b m_{2}$ shows that $z^{\prime}$ cannot be of the second colour, either. This is a contradiction to the assumption there is no monochromatic solution of equation (5).

Moreover, the total length of the interval size is at most $1+2|b|+30|b|=$ $32|b|+1$, finishing the proof of the first conclusion in the statement of the theorem.

To prove the last statement, let $W=W(a, b, c)$. For $1 \leqslant e<\frac{N}{2}$ and $\frac{N}{4 W}<$ $f<\frac{N}{2 W}$, there are $(1+o(1)) \frac{N^{2}}{8 W}$ arithmetic progressions $A_{e, f}=\{e+f t: 0 \leqslant t<$ $W\}$. By definition of $W$, each progression $A_{e, f}$ contains a monochromatic solution $(x, y, z)$ of $a x+b y+c z=0$.

On the other hand, each solution $(x, y, z)$ occurs in at most $\left(\begin{array}{c}W \\ 2\end{array}\right)$ different progressions. To see this, let $x=e+f t_{1}, y=e+f t_{2}, z=e+f t_{3}$. Given $x$ and $y$, there are at most $\left(\begin{array}{c}W \\ 2\end{array}\right)$ different choices of $t_{1}$ and $t_{2}$, and then $e, f$ and $t_{3}$ are also determined. So, there are at least $(1+o(1)) \frac{N^{2}}{8 W\left(\begin{array}{c}W \\ 2\end{array}\right)}=\left(\frac{1}{4}+o(1)\right) \frac{N^{2}}{W^{3}}$ monochromatic solutions. With $W \leqslant 32|b|+1$, the corollary follows.

From van der Waerden's Theorem one can prove the following lemma.

Lemma 6.3. Let $f: \mathbb{N} \rightarrow\{-1,1\}$ be a non-constant completely multiplicative function. Define $S^{+}=\{n: f(n)=1\}$ and $S^{-}=\{n: f(n)=-1\}$. For each positive integer $k$, there exists $N_{k}$ and $c=c(f, k)$ so that for any $N \geqslant N_{k}$, each of $S^{+} \cap[1, N]$ and $S^{-} \cap[1, N]$ contains at least $c N^{2}$ distinct $k$-term arithmetic progressions.

Proof sketch. By van der Waerden's theorem with $r=2$ colours, there is an arbitrarily long arithmetic progression in (at least) one of the two sets $S^{+}$or $S^{-}$. Multiplying the arithmetic progression with any $t$ satisfying $f(t)=-1$ allows one to swap to the other "colour class". The method of Varnavides then gives an $N$ so that at least $c^{\prime} N^{2} k$-term arithmetic progressions in one of the sets $S^{-} \cap$ $[1, N]$ or $S^{+} \cap[1, N]$, and after multiplication with $t$, one also finds $c^{\prime \prime} N^{2}$ many progressions in the other set. (One can choose $t=p_{0}$ to be the least prime with $f\left(p_{0}\right)=-1$.) 


\section{Some extremal graph theory}

Let $s \leqslant m$ and $t \leqslant n$ be positive integers, and let $V_{1}$ and $V_{2}$ be vertex sets with $\left|V_{1}\right|=m$ and $\left|V_{2}\right|=n$. Define $z(m, n ; s, t)$ to be the minimum number $z$ so that any oriented bipartite graph $\vec{G}=\left(V_{1}, V_{2}, E\right)$ with $|E|=z$ edges contains a copy of $\overrightarrow{K_{s, t}}$. The following version of a theorem by Kôvári, Sós and Turán [16] is well known (see, e.g., [1, Thm 10, p. 113]) and follows from standard double counting arguments, usually using Jensen's inequality for convex functions (the function defined by $g(x)=\left(\begin{array}{l}x \\ t\end{array}\right)$ is convex; e.g., see [1, p. 112]).

Theorem 7.1. $z(m, n, s, t) \leqslant(t-1)^{1 / s}(m-s+1) n^{1-1 / s}+(s-1) n+1$.

Below is a result that does not only guarantees one $K_{s, t}$, but many. This is proved by techniques similar to those used for Theorem 7.1 and those in [8].

Theorem 7.2. Let $\vec{G}=\left(V_{1}, V_{2}, E\right)$ be an oriented bipartite graph with $\left|V_{1}\right|=m$ and $\left|V_{2}\right|=n$, and let $s \leqslant m$ and $t \leqslant n$. Then $G$ contains at least

$$
\left(\begin{array}{c}
m \\
s
\end{array}\right)\left(\begin{array}{c}
\frac{n}{\left(\begin{array}{c}
m \\
s
\end{array}\right)}\left(\begin{array}{c}
|E| / n \\
s
\end{array}\right) \\
t
\end{array}\right)
$$

copies of $\overrightarrow{K_{s, t}}$.

Proof. For a set $S$ of vertices, let $\operatorname{deg}(S)$ denote the number of vertices adjacent to every vertex in $S$. The number of copies of $\overrightarrow{K_{s, t}}$ in $G$ is:

$$
\begin{aligned}
& \sum_{S \in\left[V_{1}\right]^{s}}\left(\begin{array}{c}
\operatorname{deg}(S) \\
t
\end{array}\right) \geqslant\left(\begin{array}{c}
m \\
s
\end{array}\right)\left(\begin{array}{c}
\operatorname{avg} \operatorname{deg}(S) \\
t
\end{array}\right) \quad \text { (by convexity) } \\
& =\left(\begin{array}{c}
m \\
s
\end{array}\right)\left(\begin{array}{c}
\frac{\sum_{S \in\left[V_{1}\right]^{s}} \operatorname{deg}(S)}{\left(\begin{array}{c}
m \\
s
\end{array}\right)} \\
t
\end{array}\right) \\
& =\left(\begin{array}{c}
m \\
s
\end{array}\right)\left(\begin{array}{c}
\frac{1}{\left(\begin{array}{c}
m \\
s
\end{array}\right)} \sum_{x \in V_{2}}\left(\begin{array}{c}
\operatorname{deg}(x) \\
s
\end{array}\right) \\
t
\end{array}\right) \\
& \geqslant\left(\begin{array}{c}
m \\
s
\end{array}\right)\left(\begin{array}{c}
\frac{1}{\left(\begin{array}{c}
m \\
s
\end{array}\right)} n\left(\begin{array}{c}
\operatorname{avg}_{V_{2}} \operatorname{deg}(x) \\
t^{2}
\end{array}\right) \\
t
\end{array}\right) \quad \text { (by convexity) } \\
& =\left(\begin{array}{c}
m \\
s
\end{array}\right)\left(\begin{array}{c}
\frac{n}{\left(\begin{array}{c}
m \\
s
\end{array}\right)}\left(\begin{array}{c}
|E| / n \\
s
\end{array}\right) \\
t
\end{array}\right) \text {. }
\end{aligned}
$$

Note that only when $\frac{n}{\left(\begin{array}{c}m \\ s\end{array}\right)}\left(\begin{array}{c}|E| / n \\ s\end{array}\right) \geqslant t$, does Theorem 7.2 guarantee at least one copy of $\overrightarrow{K_{s, t}}$.

The following simple lemma is a consequence of Stirling's formula, which states as $w \rightarrow \infty$, if $k=o\left(w^{3 / 4}\right)$, then $\left(\begin{array}{l}w \\ k\end{array}\right) \sim \frac{1}{\sqrt{2 \pi k}}\left(\frac{e w}{k}\right)^{k}$, 
Lemma 7.3. As $w \rightarrow \infty$, if $k=o\left(w^{3 / 4}\right)$, then for any $\beta \in(0,1)$,

$$
\left(\begin{array}{c}
\beta w \\
k
\end{array}\right)=(1+o(1)) \beta^{k}\left(\begin{array}{l}
w \\
k
\end{array}\right) .
$$

Using Lemma 7.3 twice helps to approximate the expression in Theorem 7.2 in certain cases:

Corollary 7.4. Let $m, n \rightarrow \infty$, and let $s=o\left(m^{3 / 4}\right), t=o\left(n^{3 / 4}\right)$. For any fixed $\beta \in(0,1)$, if $\vec{G}=\left(V_{1}, V_{2}, E\right)$ is an oriented bipartite graph with $\left|V_{1}\right|=m,\left|V_{2}\right|=n$, and $|E|=\beta m n$, the number of copies of $\overrightarrow{K_{s, t}}$ in $G$ is $(1+o(1)) \beta^{s t}\left(\begin{array}{c}m \\ s\end{array}\right)\left(\begin{array}{c}n \\ t\end{array}\right)$.

Proof. Let $\vec{G}$ satisfy the hypothesis of the theorem. By Theorem 7.2, the number of copies of $\overrightarrow{K_{s, t}}$ in $G$ is

$$
\begin{aligned}
\left(\begin{array}{c}
m \\
s
\end{array}\right)\left(\begin{array}{c}
\frac{n}{\left(\begin{array}{c}
m \\
s
\end{array}\right)}\left(\begin{array}{c}
|E| / n \\
s
\end{array}\right) \\
t
\end{array}\right) & =\left(\begin{array}{c}
m \\
s
\end{array}\right)\left(\begin{array}{c}
\frac{n}{\left(\begin{array}{c}
m \\
s
\end{array}\right)}\left(\begin{array}{c}
\beta m \\
s
\end{array}\right) \\
t
\end{array}\right) \\
& =\left(\begin{array}{c}
m \\
s
\end{array}\right)\left(\begin{array}{c}
\frac{n}{(m}(1+o(1)) \beta^{s}\left(\begin{array}{c}
m \\
s
\end{array}\right) \\
t
\end{array}\right) \\
& =\left(\begin{array}{c}
m \\
s
\end{array}\right)\left(\begin{array}{c}
(1+o(1)) n \beta^{s} \\
t
\end{array}\right) \\
& =(1+o(1)) \beta^{s t}\left(\begin{array}{c}
m \\
s
\end{array}\right)\left(\begin{array}{c}
n \\
t
\end{array}\right) .
\end{aligned}
$$

So Corollary 7.4 says that a bipartite graph containing a positive fraction of all possible edges also contains a positive fraction of all possible $K_{s, t}$-subgraphs (when $s$ and $t$ are not too large).

\section{Proof of Theorem 1.3}

The next simple lemma is a tool that can be used to assist in calculating constants from Lemma 8.4 (below).

Lemma 8.1. Let $f: \mathbb{N} \rightarrow\{-1,1\}$ be a non-constant completely multiplicative function, and let $p_{0}$ be the least prime with $f\left(p_{0}\right)=-1$. Then there exists

(a) an integer $n \leqslant 9$ such that $f(n)=f(n+1)=1$;

(b) an integer $n=p_{0}-1$ such that $f(n)=1, f(n+1)=f\left(p_{0}\right)=-1$;

(c) an integer $n \leqslant\left\lfloor p_{0}+2 \sqrt{p_{0}}\right\rfloor$ such that $f(n)=-1, f(n+1)=1$.

Proof. (a) Since $f$ is completely multiplicative, $f(1)=f(4)=f(9)=1$. If $f(2)=1$ or $f(5)=1$, we are done. If $f(2)=f(5)=-1$, then $f(10)=1$ and we are also done.

(b) This follows since $p_{0}$ is the least prime and therefore the least integer $t$ with $f(t)=-1$. 
(c) If $p_{0}=2$, then either $n=2$ or $n=3$ satisfy the condition, since $f(4)=1$. Otherwise, put $n=p_{0}$. Here $n+1$ is composed of primes with $f(p)=1$ so that $f(n+1)=1$.

Let $t^{2}$ denote the least integer square larger than $p_{0}$. Since $f\left(p_{0}\right)=-1$ and $f\left(t^{2}\right)=1$, there must be an $n \in\left[p_{0}, t^{2}-1\right]$ so that $f(n)=-1$ and $f(n+1)=1$. Since

$$
t^{2}<\left(\sqrt{p_{0}}+1\right)^{2}=p_{0}+2 \sqrt{p_{0}}+1
$$

then $n \leqslant t^{2}-1<p_{0}+2 \sqrt{p_{0}}$.

Lemma 8.2. For any positive integer $v$, all integer solutions of the equation

$$
v x_{1}-(v+1) x_{2}+x_{3}=0
$$

are of the form $\left(x_{1}, x_{2}, x_{3}\right)=(x, x+d, x+(v+1) d)$ and each such triple is a solution.

Proof. Given any solution $\left(x_{1}, x_{2}, x_{3}\right)$, define $x=x_{1}, d=x_{2}-x_{1}$ and check that $x_{3}=-v x_{1}+(v+1) x_{2}=-v x+(v+1)(x+d)=x+(v+1) d$.

Lemma 8.3. Let $f: \mathbb{N} \rightarrow\{-1,1\}$ be a non-constant completely multiplicative function and let $v \in \mathbb{Z}$. For each $i \in\{-1,1\}$, there exists a constant $c_{1}=c_{1}(f, v, i)$ so that for $N$ sufficiently large, there are at least $c_{1} N^{2}$ solutions $\left(x_{1}, x_{2}, x_{3}\right) \in$ $[1, N]^{3}$ (with the $x_{i}$ 's distinct) to equation (7) so that $f\left(x_{1}\right)=f\left(x_{2}\right)=f\left(x_{3}\right)=i$.

So Lemma 8.3 says that both $S^{-}(f)$ and $S^{+}(f)$ contain on the order of $N^{2}$ solutions (a positive fraction of all solutions) to (7). There are two easy proofs of Lemma 8.3 (the second of which serves only to show that the density result in Theorem 6.1 is not really necessary).

First proof of Lemma 8.3. By Lemma 3.1, both $S^{-}$and $S^{+}$are dense in $N$, and since the coefficients of $(7)$ sum to $v-(v+1)+1=0$, Theorem 6.1 concludes the proof.

Second proof of 8.3. By Lemma 6.3 with $k=v+2$, there are $\Theta\left(N^{2}\right)$ many $(v+2)$-term arithmetic progressions in each of $S^{-}$and $S^{+}$, and by Lemma 8.2, every solution to (7) consists of the first two terms and the last term of any such progression.

Third proof of Lemma 8.3. Apply Theorem 1.7.

Lemma 8.4. Let $f$ be a non-constant completely multiplicative function and let $\left(\eta_{1}, \eta_{2}, \eta_{3}\right) \in\{-1,1\}^{3}$. There exists a positive integer $v$ (depending only on $f$ and the $\eta_{i}$ 's) and a function $g_{v}:\left(\mathbb{Z}^{+}\right)^{2} \rightarrow\left(\mathbb{Z}^{+}\right)^{2}$ so that for each solution $(x, x+d, x+$ $(v+1) d)$ to $(7), g_{v}(x, d)=(a, b)$ satisfies

$$
f(a)=\eta_{1}, \quad f(b)=\eta_{2}, \quad f(a+b)=\eta_{3} .
$$

Furthermore, $g_{v}$ is bijective on integer solutions to (7). 
Proof. Consider all eight possible choices of $\eta_{1}, \eta_{2}, \eta_{3}$. If for some fixed choice of $\eta_{1}, \eta_{2}, \eta_{3},(a, b, a+b)$ is a solution to $(8)$, and $f(p)=-1$, then

$$
f(p a)=-\eta_{1}, \quad f(p b)=-\eta_{2}, \quad f(p a+p b)=-\eta_{3},
$$

which means that solutions to (8) need be found only when $\left(\eta_{1}, \eta_{2}, \eta_{3}\right)$ falls under one of four cases:

(a) $(1,1,1)$ or $(-1,-1,-1)$,

(b) $(1,1,-1)$ or $(-1,-1,1)$,

(c) $(1,-1,1)$ or $(-1,1,-1)$, and

(d) $(1,-1,-1)$ or $(-1,1,1)$.

Case (a): Let $v$ be the least integer with $f(v)=f(v+1)=1$. For each solution $(x, x+d, x+(v+1) d) \in\left(S^{+}\right)^{3}$ to $(7)$, put $a=v x$, and $b=x+(v+1) d$; then $a+b=$ $(v+1)(x+d), f(a)=f(v) f(x)=1, f(b)=1$, and $f(a+b)=f(v+1) f(x+d)=1$.

Case (b): Let $v$ be the least integer with $f(v)=1, f(v+1)=-1$. For each solution $(x, x+d, x+(v+1) d) \in\left(S^{+}\right)^{3}$ to $(7)$, put $a=v x, b=x+(v+1) d$; then $a+b=(v+1)(x+d), f(a)=1, f(b)=1$, and $f(a+b)=f(v+1) f(x+d)=-1$.

Case (c): Let $v$ be the least integer with $f(v)=-1, f(v+1)=1$. For each solution $(x, x+d, x+(v+1) d) \in\left(S^{+}\right)^{3}$ to $(7)$, put $a=x+(v+1) d, b=v x$; then $a+b=(v+1)(x+d), f(a)=1, f(b)=-1$, and $f(a+b)=1$.

Case (d): Interchange $a$ and $b$ from Case (c): Let $v$ be the least integer with $f(v)=-1, f(v+1)=1$. For each solution $(x, x+d, x+(v+1) d) \in\left(S^{+}\right)^{3}$ to $(7)$, put $a=v x, b=x+(v+1) d$; then $a+b=(v+1)(x+d), f(a)=-1, f(b)=1$, and $f(a+b)=1$.

Note that by Lemma 8.1, in all four cases $v \leqslant \max \left(9, p_{0}+2 \sqrt{p_{0}}\right)$.

Summarizing, for each fixed $v$, the function $g_{v}$ that bijectively maps solutions of (7) to triples $(a, b, a+b)$ is given by, in cases (a), (b), and (d),

$$
\left.g_{v}((x, d))=(v x, x+(v+1) d,(v+1)(x+d))\right)
$$

or in case (c),

$$
g_{v}((x, d))=(x+(v+1) d, v x,(v+1)(x+d)) .
$$

Lemmas 8.3 and 8.4 now immediately give:

Corollary 8.5. If $f$ is a non-constant completely multiplicative function, for each $\left(\eta_{1}, \eta_{2}, \eta_{3}\right) \in\{-1,1\}^{3}$, there exists a constant $c_{3}=c_{3}\left(f, \eta_{1}, \eta_{2}, \eta_{3}\right)$ so that for sufficiently large $N$ there are at least $c_{3} N^{2}$ solutions to (8).

Here $c_{3}=\frac{1}{138,000(v+1)^{3}}$ is an admissible value, in view of $|b|=v+1$ and Theorem 1.7.

Finally, we come to the proof of the main theorem. Having worked out all the necessary bits separately, the proof is short. 
Proof of Theorem 1.3. For each of the eight cases $\left(\eta_{1}, \eta_{2}, \eta_{3}\right) \in\{-1,1\}^{3}$ define a bipartite graph $G=\left(V_{1} \cup V_{2}, E\right)$ as follows:

$$
\begin{aligned}
V_{1} & =\left\{i \in[1, N]: f(i)=\eta_{1}\right\}, \\
V_{2} & =\left\{j \in[1, N]: f(j)=\eta_{2}\right\}, \\
E & =\left\{(i, j) \in V_{1} \times V_{2}: f(i+j)=\eta_{3}\right\} .
\end{aligned}
$$

By Lemma 3.1, in each of the eight cases, $\left|V_{1}\right| \geqslant c_{1} N,\left|V_{2}\right| \geqslant c_{2} N$ and by Corollary $8.5,|E| \geqslant c_{3} N^{2}$. Note that the constants depend on $f$, and in particular, on the size of the least prime $p_{0}$ with $f\left(p_{0}\right)=-1$.

Sets of integers $\mathcal{A}, \mathcal{B} \subseteq[1, N]$ with $f(a)=\eta_{1}, f(b)=\eta_{2}$, and $f(a+b)=\eta_{3}$ (for all $a \in \mathcal{A}, b \in \mathcal{B}$ ), correspond to a complete bipartite subgraph $K_{s, t}$ of $G$ with $s=|\mathcal{A}|, t=|\mathcal{B}|$. By Corollary 7.4, with $|E| \geqslant \frac{c_{3}}{c_{1} c_{2}}\left|V_{1}\right| \cdot\left|V_{2}\right|, G$ contains at least $\left(c_{4}+o(1)\right)^{s t}\left(\begin{array}{c}m \\ s\end{array}\right)\left(\begin{array}{c}n \\ t\end{array}\right)$ copies of $K_{s, t}$. So with $c_{4}=\frac{c_{3}}{c_{1} c_{2}}$ the theorem follows.

Acknowledgements. The first author was partially supported by Royal Holloway (University of London), the University of Manitoba, the Finite Structures project, in the framework of the European Community's "Structuring the European Research Area" programme and the Austrian-French Amadée Program FR03/2012 and would like to thank Miki Simonovits for discussions.

The second author was partially supported by NSERC grant 228064-09.

\section{References}

[1] B. Bollobás, Modern graph theory, Graduate texts in mathematics 184, Springer, New York, 1998.

[2] Y. Buttkewitz, C. Elsholtz, Patterns and complexity of multiplicative functions, J. London Math. Soc. (2) 84 (2011), 578-594.

[3] J. Cassaigne, S. Ferenczi, C. Mauduit, J. Rivat, and A. Sárközy, On finite pseudorandom binary sequences III: The Liouville function, I, Acta Arith $\mathbf{8 7}$ (1999), 367-390.

[4] S. Chowla, The Riemann hypothesis and Hilbert's tenth proble $m$, New York - London - Paris, Gordon and Breach (1965).

[5] E.S. Croot, On the oscillations of multiplicative functions taking values \pm 1 , J. Number Theory 98 (2003), 184-194.

[6] H. Daboussi and A. Sárközy, On pseudorandom properties of multiplicative functions, Acta Math. Hungar. 98 (2003), 273-300.

[7] C. Elsholtz, Triples of primes in arithmetic progressions, (English summary), Q. J. Math. 53 (2002), no. 4, 393-395.

[8] P. Erdôs, On extremal problems of graphs and generalized graphs, Israel J. Math. 2 (1964), 183-190.

[9] P. Frankl, R.L. Graham, V. Rödl, Quantitative theorems for regular systems of equations, J. Combin. Theory Ser. A 47 (1988), 246-261. 
[10] Z. Füredi, Turán type problems, in Surveys in Combinatorics, 1991, (ed. A. D. Keedwell), London Math. Soc. Lecture Notes 166, Cambridge University Press, Cambridge (1991), 253-300.

[11] R. Graham, V. Rödl, A. Ruciński, On Schur properties of random subsets of integers, J. Number Theory 61 (1996), 388-408.

[12] S.W. Graham, D. Hensley, Problem E3025, Amer. Math. Monthly 90 (1983), 707.

[13] K. Gyarmati, On divisibility properties of integers of the form ab +1 , Period. Math. Hungarica 43 (2001), 71-79.

[14] G. Harman, J. Pintz, D. Wolke, A note on the Möbius and Liouville functions, Studia Sci. Math. Hungar. 20 (1985), 295-299.

[15] A. Hildebrand, On consecutive values of the Liouville function, Enseign. Math. (2) 32 (1986), 219-226.

[16] T. Kôvári, V.T. Sós and P. Turán, On a problem of K. Zarankiewicz, Colloq. Math. 3 (1954), 50-57.

[17] K. Matomäki, M. Radziwiłł, Multiplicative functions in short intervals, http://arxiv.org/abs/1501.04585.

[18] K. Matomäki, M. Radziwiłł, T. Tao, An averaged form of Chowla's conjecture, http://arxiv.org/abs/1503.05121.

[19] P.A. Parrilo, A. Robertson, D. Saracino, On the asymptotic minimum number of monochromatic 3-term arithmetic progressions, J. Combin. Theory Ser. A 115 (2008), 185-192,

[20] J. Rivat, A. Sárközy, C.L. Stewart, Congruence properties of the $\Omega$-function on sumsets, Illinois J. Math. 43 (1999), 1-18.

[21] K.F. Roth, Sur quelques ensembles d'entiers, C.R. Acad. Sci. Paris 234 (1952), 388-390.

[22] K.F. Roth, On certain sets of integers, J. London Math. Soc. 28 (1953), 104-109.

[23] A. Schinzel, W. Sierpiński, Sur certaines hypothèses concernant les nombres premiers, Acta Arith. 4 (1958), 185-208; Corrigendum: ibid. 5 (1959), 259.

[24] E. Szemerédi, On sets of integers containing no $k$ elements in arithmetic progression, Acta Arith. 27 (1975), 199-245.

[25] G. Tenenbaum, Introduction to analytic and probabilistic number theory, Cambridge studies in advanced mathematics 46, Cambridge University Press, 1995.

[26] A. Thomason, On finite Ramsey numbers, European J. Combin. 3 (1982), 263-273.

[27] B.L. van der Waerden, Beweis einer Baudetschen Vermutung, Nieuw. Arch. Wisk. 15 (1927), 212-216.

[28] P. Varnavides, On certain sets of positive density, J. London Math. Soc. 30 (1959), 358-360.

[29] E. Wirsing, Das asymptotische Verhalten von Summen über multiplikative Funktionen II, Acta Math. Acad. Sci. Hungar. 18 (1967), 411-467. 
Addresses: Christian Elsholtz: Institut für Analysis und Computational Number Theory, Technische Universität Graz, Steyrergasse 30, A-8010 Graz, Austria;

David S. Gunderson: Department of Mathematics, University of Manitoba, Winnipeg, MB, Canada, R3T 2N2.

E-mail: elsholtz@math.tugraz.at, gunderso@cc.umanitoba.ca

Received: 23 April 2014; revised: 6 May 2014 